\title{
Learning about time within the spinal cord: evidence that spinal neurons can abstract and store an index of regularity
}

\author{
Kuan H. Lee ${ }^{1 *}$, Joel D. Turtle ${ }^{2}$, Yung-Jen Huang ${ }^{2}$, Misty M. Strain ${ }^{2}$, Kyle M. Baumbauer ${ }^{3}$ \\ and James W. Grau ${ }^{2}$ \\ ${ }^{1}$ Department of Neurobiology, Center for Pain Research, University of Pittsburgh School of Medicine, Pittsburgh, PA, USA, \\ ${ }^{2}$ Department of Psychology, Cellular and Behavioral Neuroscience, Texas A\&M University, College Station, TX, USA, ${ }^{3}$ School \\ of Nursing, University of Connecticut, Storrs, CT, USA
}

Prior studies have shown that intermittent noxious stimulation has divergent effects on spinal cord plasticity depending upon whether it occurs in a regular (fixed time, FT) or irregular (variable time, VT) manner: In spinally transected animals, VT stimulation to the tail or hind leg impaired spinal learning whereas an extended exposure to FT stimulation had a restorative/protective effect. These observations imply that lower level systems are sensitive to temporal relations. Using spinally transected rats, it is shown that the restorative effect of FT stimulation emerges after 540 shocks; fewer shocks generate a learning impairment. The transformative effect of $\mathrm{FT}$ stimulation is related to the number

OPEN ACCESS

Edited by:

Regina Marie Sullivan, Nathan Kline Institute and NYU

School of Medicine, USA

Reviewed by:

Ray de Leon,

California State University, USA

Jenny Haefeli,

University of California,

San Francisco, USA

${ }^{*}$ Correspondence:

Kuan H. Lee

millylee@pitt.edu

Received: 04 August 2015 Accepted: 28 September 2015

Published: 21 October 2015

Citation:

Lee KH, Turtle JD, Huang Y-J, Strain MM, Baumbauer KM and Grau JW (2015) Learning about time within the spinal cord: evidence that spinal neurons can abstract and store an index of regularity.

Front. Behav. Neurosci. 9:274. doi: 10.3389/fnbeh.2015.00274 of shocks administered, not the duration of exposure. Administration of $360 \mathrm{FT}$ shocks induces a learning deficit that lasts $24 \mathrm{~h}$. If a second bout of FT stimulation is given a day after the first, it restores the capacity to learn. This savings effect implies that the initial training episode had a lasting (memory-like) effect. Two bouts of shock have a transformative effect when applied at different locations or at difference frequencies, implying spinal systems abstract and store an index of regularity (rather than a specific interval). Implications of the results for step training and rehabilitation after injury are discussed.

Keywords: spinal cord, time, instrumental learning, memory, central pattern generator

\section{INTRODUCTION}

Over the last few decades, our view of spinal cord function has evolved. It is now recognized that sensory input can induce lasting alterations within the spinal cord that transform the response elicited by subsequent stimuli (for reviews, see Grau et al., 2006, 2012, 2014). For example, pain (nociceptive) signals can induce a lasting increase in neural excitability within the spinal cord (central sensitization) that enhances behavioral reactivity and pain (Sandkühler, 2000; Willis, 2001). These neural modifications depend upon neurochemical systems (e.g., the NMDA receptor, NMDAR) analogous to those implicated in brain-dependent learning and memory (Woolf and Thompson, 1991; Ji et al., 2003). Likewise, behavioral experience can engage motor programs within the spinal cord that promote the re-acquisition of hindlimb stepping (Barbeau and Rossignol, 1987; Bélanger et al., 1996; Edgerton et al., 2004; Barrière et al., 2008). 
We have sought to characterize how and when a behavioral experience affects spinal cord function (Grau et al., 2006; Grau, 2014). We have shown that neurons within the lumbosacral spinal cord are sensitive to response-outcome (instrumental) relations and can support learning after communication with the brain has been surgically transected (Grau et al., 1998). In this preparation, transected rats are given electrical stimulation (shock; the outcome) to the tibialis anterior muscle of one hind leg whenever the leg is extended (the response). This response-outcome relation produces a gradual increase in flexion duration that minimizes shock exposure. Importantly, if one group (Master) of rats is given response-contingent (controllable stimulation) shock while another (Yoked) receives shock independent of leg position (uncontrollable stimulation), only the Master subjects exhibit an increase in flexion duration. When these subjects are later tested under common conditions with controllable shock (applied to the same or contralateral leg), Master rats learn more rapidly whereas Yoked subjects exhibit a learning impairment. The learning deficit observed after uncontrollable stimulation can be induced by variable intermittent shock applied to the leg or tail and lasts up to $48 \mathrm{~h}$ (Crown et al., 2002). This phenomenon is of clinical interest because it has been related to the induction of central sensitization and has been shown to impact recovery after a contusion injury (Grau et al., 2004; Ferguson et al., 2006).

We recently discovered that the long-term effect of intermittent nociceptive stimulation is modulated by temporal variables as well as behavioral control (Baumbauer et al., 2008, 2009, 2012; Baumbauer and Grau, 2011). The first indication of this emerged from a study examining the effect of electrophysiological stimulation of the sciatic nerve. We had shown that exposure to 180-900 intermittent shocks (80 $\mathrm{ms}$ ), presented in a variable manner (on a rectangular distribution that varied between 0.2 and $3.8 \mathrm{~s}$ (mean $=2 \mathrm{~s}$; $0.5 \mathrm{~Hz}$ )) induces a lasting learning impairment (Baumbauer et al., 2008). To explore how the frequency of stimulation affected the induction of this effect, we fixed the interval between shocks. Under these conditions, 180 shocks given in a regular manner (fixed time, FT) induced a learning impairment, but 900 shocks did not. It appeared that continued exposure to regular stimulation restores the capacity to learn.

Prior work had shown that training with controllable stimulation can both prevent, and reverse, the learning impairment induced by variable intermittent shock (Crown and Grau, 2001). Given this, we examined whether exposure to regular shock (720 FT shocks presented at $0.5 \mathrm{~Hz}$ ) could prevent, and restore, the learning impairment induced by 180 shocks given on a variable time (VT) schedule. We found that FT stimulation had a protective/restorative effect analogous to that observed with behavioral control (Baumbauer et al., 2009). Furthermore, the protective effect of FT stimulation lasts at least $24 \mathrm{~h}$ and depends upon a form of NMDAR-mediated plasticity and protein synthesis (Baumbauer et al., 2009). Like behavioral control, exposure to FT stimulation appears to re-establish the capacity to learn by up-regulating the expression of brain-derived neurotrophic factor (BDNF).
The present study seeks to clarify the conditions under which FT stimulation has a transformative effect that re-establishes the capacity to learn (the FT effect). We then use this information to explore what is learned. A hallmark of learning is that it has a lasting effect, laying down a kind of memory that preserves key features of the learning episode, producing a savings effect that allows the neural machinery to span gaps in time. If the process that abstracts regularity reflects a kind of learning, it should exhibit a form of savings, allowing the beneficial effect of FT stimulation to accrue when training is broken into chunks and given across days. Supporting this, we show that a single bout of 360 FT shocks produces a lasting learning impairment and that a second bout, given 24 $\mathrm{h}$ later, restores the capacity to learn. We then examine what is learned during the initial bout of stimulation that allows a subsequent bout of FT shock to have a transformative effect. Our results suggest that the critical feature is linked to the regularity of the stimulation, not the specific inter-stimulus interval (ISI).

\section{MATERIALS AND METHODS}

\section{Subjects}

Subjects were male Sprague-Dawley rats obtained from Harlan (Houston, TX, USA) that were approximately 100-120 days old and weighed between 300 and $400 \mathrm{~g}$. All subjects were pair housed and maintained on a $12 \mathrm{~h}$ light/dark cycle, with all behavioral testing performed during the light cycle. Food and water were available ad libitum. All experiments were carried out in accordance with National Institutes of Health (NIH) standards for the care and use of laboratory animals (NIH publications No. 80-23), and were approved by the University Laboratory Animal Care Committee at Texas A\&M University. Every effort was made to minimize suffering and limit the number of animals used.

\section{Spinal Cord Transection}

Before surgery, the fur over the surgical site was shaved and disinfected with betadine solution (H-E-B, San Antonio, TX, USA). Subjects were anesthetized with isoflurane gas. Anesthesia was induced at $5 \%$ isoflurane and maintained at $2-3 \%$ isoflurane. Each subject's head was rendered immobile in a stereotaxic apparatus, and a small $(5.0 \times 4.0 \times 2.5 \mathrm{~cm})$ gauze pillow was placed under the subject's chest to provide support for respiration.

To perform a transection at the second thoracic vertebra (T2), an anterior to posterior incision was made and the tissue just rostral to T2 was cleared using rongeurs, and the cord exposed and cauterized. The remaining gap in the cord was filled with Gelfoam (Pharmacia Corp., Kalamazoo, MI, USA) and the wound was closed with Michel clips (Fisher Scientific, Waltham, MA, USA).

Following closure of the wound on the back, the surface of both legs were shaved for electrode placement. Intraperitoneal injections $(3 \mathrm{~mL})$ of $0.9 \%$ saline solution were administered postoperatively to prevent dehydration. Following surgery, rats were 
placed in a temperature-controlled environment $\left(25.5^{\circ} \mathrm{C}\right)$ and monitored until awake. All rats were checked every 6-8 h during the $18-24 \mathrm{~h}$ post-surgical period. During this time, hydration was maintained with supplemental injections of saline, and the rats' bladders and colons were manually expressed as needed.

Spinal transections were confirmed by: (a) inspecting the cord at the time of surgery; (b) observing the behavior of the subjects after they recovered to ensure that they exhibited paralysis below the level of the forepaws and did not exhibit any supraspinallymediated pain responses to leg shock; and (c) by examining the tissue postmortem.

\section{Stimulation Procedures}

Stimulation was applied while subjects were loosely restrained in Plexiglas tubes $(23.5 \mathrm{~cm}$ long $\times 8 \mathrm{~cm}$ internal diameter). The front of each tube was sealed, and the tubes were painted black, providing a dark enclosure in which rats could rest undisturbed. Holes were drilled into the anterior portion of the tubes to allow for ventilation. Two slots were cut $4 \mathrm{~cm}$ apart and $1.5 \mathrm{~cm}$ from the posterior end of the tube to allow both hind legs to hang freely.

Tail shock was administered through electrodes constructed from a modified fuse clip. The electrode was coated with Spectra electrode gel (Harvard Appartus, Holliston, MA, USA) and secured with tape approximately $5 \mathrm{~cm}$ from the base of the tail. All subjects were loosely restrained in the Plexiglas tubes described above. A constant current $1.5 \mathrm{~mA}$ shock was delivered using a 660-V transformer with shock onset and offset controlled by the computer.

Leg shock was administered to the tibialis anterior muscle. Prior to testing, the area over the muscle was shaved. An electrode constructed from a stainless steel wire $\left(0.05 \mathrm{~mm}^{2}\right.$ [30 AWG]) was inserted through the skin over the tibia, $1.5 \mathrm{~cm}$ from the tarsus. A second electrode made from a fine wire $\left(0.01 \mathrm{~mm}^{2}\right.$ [36 AWG], magnet wire single beldsol) was inserted perpendicular to the leg, through the body of the tibialis anterior muscle, $1.7 \mathrm{~cm}$ above the first electrode. The electrodes were connected to a constant current AC shock generator (Model SG-903; BRS/LVE, Laurel MD) and shock intensity was adjusted to a level that produced a $0.4 \mathrm{~N}$ flexion response, as described in Grau et al. (1998).

Custom software running on a Macintosh computer was used to control the presentation of shock. FT shocks were 80 $\mathrm{ms}$ in duration and occurred at a regular interval (a fixed ISI) that was typically set to $2 \mathrm{~s}(0.5 \mathrm{~Hz})$. VT shocks of the same duration were presented using a variable ISI that ranged from 0.2 to $3.8 \mathrm{~s}$ (rectangular distribution) with a mean of $2 \mathrm{~s}$.

\section{Instrumental Testing}

Testing was conducted while subjects were loosely restrained in the Plexiglas tubes. Both hindlegs were freely hanging over a salt bath $(\mathrm{NaCl})$. Leg shock was delivered to the tibials anterior muscle as described above. A contact electrode was constructed from a $7 \mathrm{~cm}$ long, 0.46 diameter, stainless steel rod. The contact electrode was taped to the plantar surface of the rat's foot (Orthaletic, $1.3 \mathrm{~cm}$ [width]; Johnson and Johnson, New Brunswick, NJ) with the end positioned directly in front of the plantar protuberance. Heatshrink tubing electrically insulated the rod from the paw. A fine wire $\left[0.01 \mathrm{~mm}^{2}\right.$ (36 AWG), magnet wire single beldsol] was attached to the end of the rod at a point under the insulation. This wire extended from the rear of the foot and was connected to a digital input board that was monitored by the Macintosh computer. To minimize lateral leg movements, a piece of porous tape (Orthaletic, $1.3 \mathrm{~cm}$ [width]) was wrapped around the leg above the tarsus and attached under the front panel of the restraining tube. A rectangular plastic dish $(11.5 \mathrm{~cm}$ $[\mathrm{w}] \times 19 \mathrm{~cm}[\mathrm{l}] \times 5 \mathrm{~cm}[\mathrm{~d}])$ was positioned $7.5 \mathrm{~cm}$ below the restraining tube and filled with a $\mathrm{NaCl}$ solution with a drop of soap to reduce surface tension. A ground wire was connected to a $1 \mathrm{~mm}$ wide stainless steel rod, which was placed in the solution. Three short $(0.15 \mathrm{~s})$ shock pulses were applied and the level of the salt solution was adjusted so that the tip of the contact electrode was submerged $4 \mathrm{~mm}$ below the surface. Subjects then received $30 \mathrm{~min}$ of response contingent shock (instrumental testing). When the contact electrode touched the underlying salt solution, shock was delivered to the tibialis anterior muscle causing the ankle to flex, lifting the contact electrode out of the salt solution.

Leg position was monitored using a Macintosh computer at a sampling rate of $30 \mathrm{~Hz}$. Performance was measured over time in $30,1 \mathrm{~min}$ time bins. The computer monitoring leg position recorded an increase in response number whenever the contact electrode was raised above the salt solution. Response duration was derived from time in solution and response number in $1 \mathrm{~min}$ time bins using the following equation: Response Duration = $(60 \mathrm{~s}-$ time in solution) $/($ Response Number +1$)$. Subjects capable of instrumental learning exhibit a progressive increase in response duration that minimizes net shock exposure (Grau et al., 1998).

To evaluate whether our experimental treatment affected baseline behavioral reactivity, we analyzed both the shock intensity required to elicit a flexion force of $0.4 \mathrm{~N}$ and the duration of the first shock-elicited flexion response. Independent analysis of variances (ANOVAs) showed that there were no group differences on either measure across all experiments, $F^{\prime}$ s $<2.58, p>0.05$.

\section{Statistics}

All data were analyzed using repeated measures ANOVA. When necessary, post hoc comparisons of the group means were performed using Duncan's New Multiple Range test. In all cases, a criterion of $p<0.05$ was used to judge statistical significance.

\section{General Experimental Design}

All subjects received a complete spinal transection at the second thoracic level. Twenty-four hours after surgery, transected subjects were exposed to electrical shock applied to the tail. Experiment 1 varied the number of fixed spaced shocks administered (180, 360, 540, 720, 900 [0.5 Hz] FT shocks). Experiment 2 examined the effect of extended exposure (4500 shocks, $0.5 \mathrm{~Hz}$ ) to fixed spaced or variable spaced shock, 900 
VT shocks, 4500 VT shocks). In both experiments, subjects were tested for performance on an instrumental learning task immediately after tailshock. Experiment 3 varied the number of shocks (360 or 720 FT shocks) as a function of time across 6,12 or $24 \mathrm{~min}$. Instrumental learning was tested $24 \mathrm{~h}$ later. Experiment 4 spaced FT stimulation [0.5 Hz] across two days, with subjects receiving 360 FT shocks or no shocks on Day 1, and 360 FT shocks or no shocks on Day 2. Finally, Experiment 5 again divided the period of stimulation across two days, but varied whether the frequency remained the same or differed (at 0.5 or $2 \mathrm{~Hz}$ ). For both Experiment 4 and 5, subjects were instrumentally tested after shock treatment on Day 2.

\section{RESULTS}

\section{Experiment 1: Restorative Effect of FT Stimulation Emerges After $\mathbf{5 4 0}$ Shocks}

From past work we know that exposure to 180 shocks at 0.5 $\mathrm{Hz}$ induces a learning impairment in spinally transected rats whether the stimuli occur in a regular or irregular manner (Baumbauer et al., 2008, 2009). However, when shock number is increased 4-5 fold (720-900 shocks), only variable stimulation impaired learning; regular stimulation eliminated the learning impairment and inducing a restorative effect that counters the adverse effect of variable shock (Baumbauer et al., 2009). In subsequent experiments, we use the recovery of learning to assay how spinal systems time. To do so, we need a more precise map of when regularity matters. The present experiment addresses this issue by determining when the restorative effect of FT stimulation emerges (with shock frequency held constant at $0.5 \mathrm{~Hz}$ ).

The experiment used 40 rats $(n=8)$ with the experimental design detailed in the top panel of Figure 1. Twenty-four hours after a T2 transection, subjects were placed in the restraining tubes and tail electrodes were attached. All subjects were restrained for $30 \mathrm{~min}$. During this period, subjects received $180,360,540,720$ or 900 FT tail shocks spaced 2 s apart. The onset of stimulation was delayed for subjects that received less than 900 FT shocks, to equate the interval between the last shock and the onset of testing. Subjects were then setup for instrumental testing and received $30 \mathrm{~min}$ of responsecontingent leg shock. Testing was conducted using either the left or right leg and this was counter-balanced across groups.

The effect of shock number on response duration is depicted in Figure 1A. Subjects given 360 or fewer shocks exhibited a learning deficit, replicating previous results (Baumbauer et al., 2008, 2009). Subjects given 540 or more shocks were able to learn when tested with controllable shock. An ANOVA revealed a main effect of shock number $F_{(4,35)}$ $=13.15, p<0.001$. Also, the Time $\times$ Shock number interaction was significant, $F_{(29,1015)}=1.76, p<0.001$. Post hoc comparisons of the group means (Figure 1A) confirmed that subjects given 180 and 360 stimulations differed from those that received 540, 720 and 900 stimulations $(p<0.05)$. No other comparisons were significant $(p>$ 0.05).

As in past studies, subjects that failed to learn exhibited the highest rate of responding (Figure 1B). An ANOVA showed that shock number affected the rate of responding, $F_{(4,35)}=5.80$, $p<0.01$. Post hoc comparisons of the group means showed that the groups that received 180 or 360 shocks differed from those that received 540 or more $(p<0.05)$. No other comparisons were significant $(p>0.05)$.

As previously reported, 180 fixed spaced shocks induced a learning impairment (Baumbauer et al., 2008, 2009). Continued exposure to fixed spaced stimulation eliminated this effect. The transition between these effects appears to occur after 360 shocks, with a minimum of 540 . As observed in earlier studies, learning was accompanied by a reduction in the rate of responding (Grau et al., 1998, 2006). Indeed, the two groups that failed to learn (as indexed by an increase in flexion duration) exhibited the highest rate of responding. This is important because it shows that the failure to learn does not reflect a performance deficit. Because a similar inverse relation between response duration and number was observed in all of the subsequent experiments, and because the former provides a more straight-forward measure of learning (Grau et al., 1998), only response duration is presented in subsequent experiments.

\section{Experiment 2: Additional Stimulation Produces a Similar Result}

Our assumption is that continued exposure to fixed spaced stimulation engages a qualitatively distinct process that transforms how spinal circuits function, restoring the capacity to learn and countering the maladaptive effects of variable shock (Baumbauer et al., 2008, 2009). It is implicitly assumed here that this transformation depends upon stimulus regularity-that only FT stimulation has a restorative effect. It could be argued, however, that the difference between regular and irregular stimulation is quantitative in nature, not qualitative. From this perspective, both VT and FT stimulation should have a non-monotonic effect, with a lower number of stimuli impairing plasticity and a higher number restoring the capacity for learning. For example, this could occur because stimuli separated by roughly $2 \mathrm{~s}$ are particularly effective at producing the protective effect. If it is then assumed that the relative effectiveness of this training interval is normally distributed, any distribution that includes these values (including VT stimulation) should produce a restorative effect, albeit less rapidly. We evaluated this possibility by comparing the effect of FT and VT stimulation when shock number is increased five fold (to 4,500 shocks).

Forty rats underwent a spinal transection and were randomly assigned to one of five conditions $(n=8)$ as indicated at the top of Figure 2. A day after surgery, subjects were placed in the restraining tubes for a period of $150 \mathrm{~min}$ and had the tail electrodes attached. One group received no shock (Unshocked). The remaining subjects received either 900 or 4,500 tail 

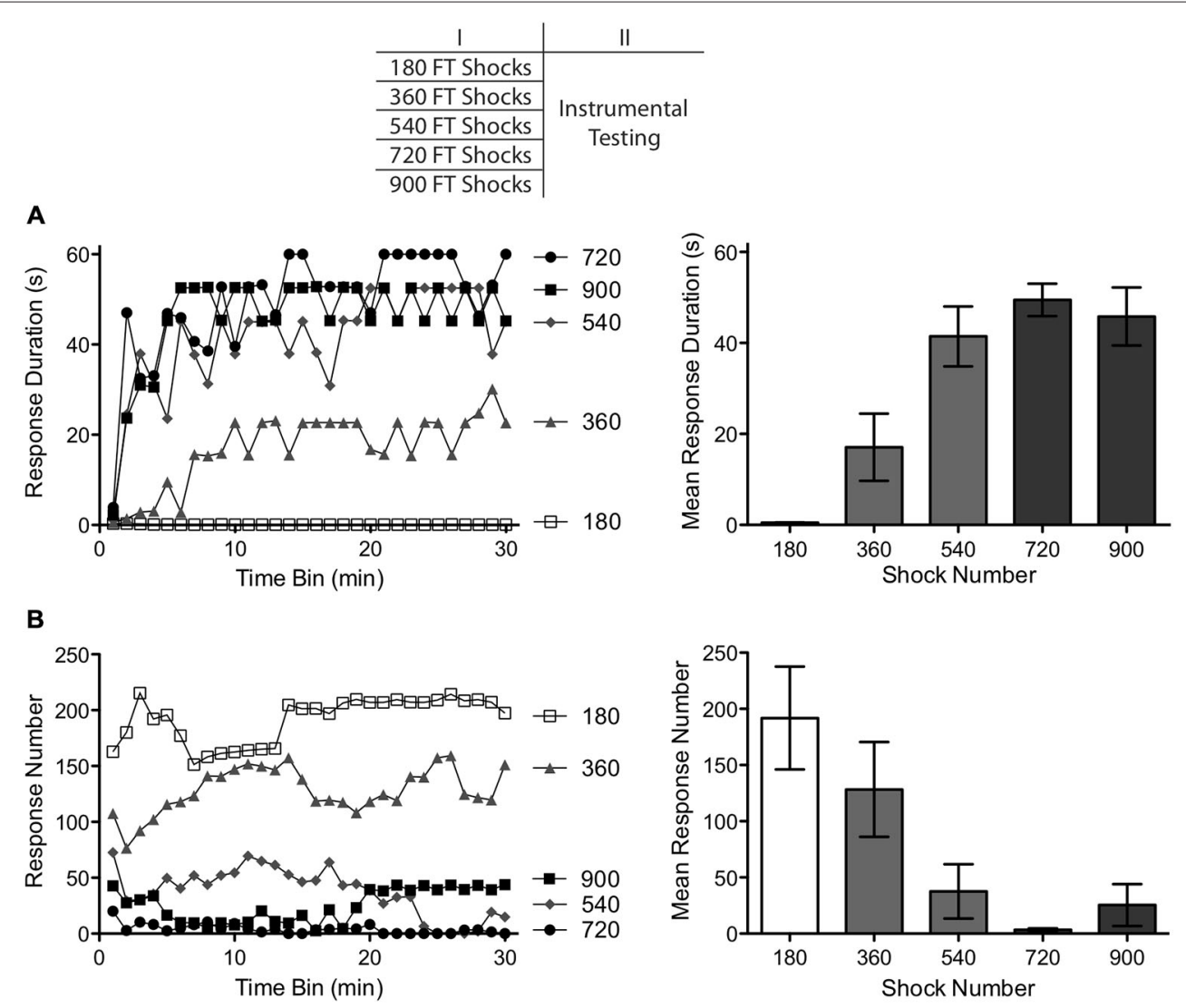

FIGURE 1 | Impact of varying numbers of fixed time (FT) shocks (180-900) on instrumental learning. A day after spinal transection, subjects received FT shocks (180-900) and were then tested with response-contingent leg shock applied to one hind leg. (A) Subjects that had previously received 540 or more shocks exhibited a progressive increase in response duration over the course of the 30 min test period (top left). Subjects that received fewer shocks (180-360) exhibited poor learning. (B) Rats that failed to learn also exhibited the highest rates of responding (bottom left). The panels to the right illustrate mean performance, collapsed across the 30 min of testing and the error bars depict the \pm SEM.

shocks at $0.5 \mathrm{~Hz}$ on a FT or VT ISI. To equate the interval between the last shock and testing, subjects given 900 shocks received the stimulation during the last $30 \mathrm{~min}$ of restraint. Instrumental learning was then tested for $30 \mathrm{~min}$ as described above.

We found that FT and VT stimulation had divergent effects on spinal learning independent of whether subjects received 900 or 4,500 shocks (Figure 2). An ANOVA confirmed that the main effect of shock treatment was significant, $F_{(4,25)}=5.46, p<$ 0.005 . There was also a significant effect of time, and Time $\times$ Shock Treatment interaction, both F's $>1.52, p<0.001$. Post hoc comparisons of the group means showed that the two groups that received variable shock differed from the unshocked group and the two FT treated groups $(p<0.05)$. No other comparison was significant $(p>0.05)$.

If VT stimulation has a non-monotonic effect on learning, increasing shock number should transform how it affects spinal function and reinstate the capacity to learn. No evidence for this was obtained. Independent of the number of shocks presented (900 or 4,500), VT stimulation induced a learning impairment. This suggests that the difference between VT and FT stimulation is not simply quantitative in nature. Rather, exposure to FT stimulation appears to have a qualitatively distinct effect.

\section{Experiment 3: Shock Number, not the Duration of Exposure, is Critical}

Experiment 1 showed that exposure to 540-900 fixed spaced shock re-established the capacity for instrumental learning, a hallmark of the FT effect. In subsequent experiments, we use this finding to explore the processes that underlie learning about time. To do so, we compare the effect of a sub-threshold level of stimulation (360 shocks) to the effect of 720 shocks. The latter value is used because it has been shown to have a lasting restorative effect and it is a multiple of 360 , which simplifies our analyses of additivity.

In evaluating the effect of shock number, we have held shock frequency constant (at $0.5 \mathrm{~Hz}$ ). As a result, shock number was confounded with duration of exposure. It is therefore possible that 360 and 720 shocks have distinct effects because the latter occurs over a period of time $(24 \mathrm{~min})$ that is twice as long. To address this possibility, we compared the effect of 360 vs. 720 shocks given over a period of 6,12 or $24 \mathrm{~min}$. If the emergence 


\begin{tabular}{c|c} 
I & $\|$ \\
\hline 900 FT Shocks & \\
\cline { 1 - 1 } 900 VT Shocks & Instrumental \\
\cline { 1 - 1 } 4,500 FT Shocks & Testing \\
\cline { 1 - 1 } 4,500 VT Shocks &
\end{tabular}
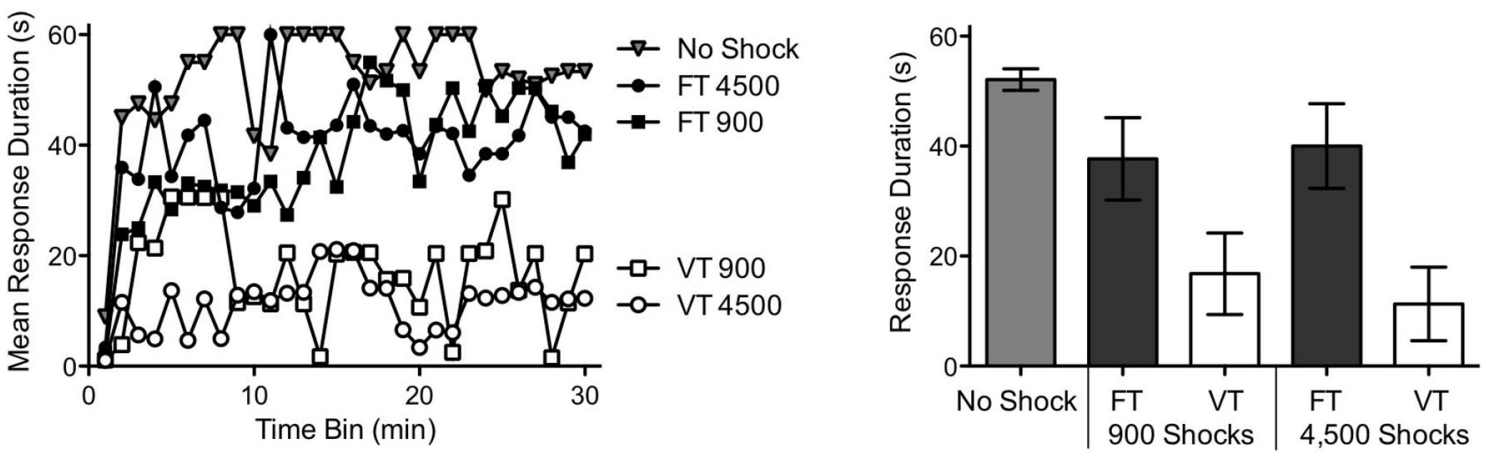

FIGURE 2 | Relative impact of 900-4,500 shocks, given in a fixed (FT) or variable (VT) manner, on instrumental learning. Spinally transected rats received 900 FT or VT shocks, 4,500 FT or VT shocks, or no shock. Instrumental learning was then tested for 30 min. Subjects that received no shock or FT shock exhibited a progressive increase in response duration indicative of learning. Subjects that received 900-4,500 VT shocks failed to learn. The top panel depicts the design of the experiment. Performance over the 30 min test period is illustrated in the left panel and mean performance is depicted to the right. The error bars depict the \pm SEM.

of the FT effect is tied to shock number, duration of exposure should not matter. Conversely, if the critical variable is the duration of exposure, 24 min of stimulation should reinstate the capacity to learn independent of whether subjects received 360 or 720 shocks.

This experiment used 48 subjects $(n=8)$. A day after the spinal transection, subjects were placed in restraining tubes and had tail electrodes attached as described above. They then received 360 or 720 tail shocks given over a period of 6, 12 or $24 \mathrm{~min}$ (Figure 3). All subjects were restrained for an equivalent period of time (24 min). To verify that stimulus exposure had a long-term effect on spinal function, instrumental testing was conducted $24 \mathrm{~h}$ later. Subjects were tested with $30 \mathrm{~min}$ of response-contingent shock as described above. The top panel of Figure 3 details the experimental design.

Exposure to 360 fixed spaced shocks induced a learning impairment independent of whether the shocks were presented over a period of $6(1.0 \mathrm{~Hz}), 12(0.5 \mathrm{~Hz})$ or $24 \min (0.25 \mathrm{~Hz})$ (Figure 3). Conversely, rats given additional shocks (720) were able to learn independent of whether the stimuli were given over a period of $6(2 \mathrm{~Hz}), 12(1.0 \mathrm{~Hz})$ or $24 \mathrm{~min}(0.5 \mathrm{~Hz})$. An ANOVA revealed a main effect of shock number, $F_{(1,42)}=23.81, p<0.001$. Neither the main effect of session duration, nor the interaction between session duration and shock number, were significant, Fs $<1.63, p<0.5$. Post hoc comparisons confirmed that the groups that received 360 stimulations were significantly different from the groups that received 720 stimulations $(p<0.05)$. No other group difference was significant $(p>0.05)$.

Within the frequency range tested, the emergence of the restorative effect of FT stimulation was determined by the number of shocks given, not the duration of exposure. Outside of these bounds, a different pattern could emerge. In part, this is because the learning impairment is most robust within a frequency range of $0.1-2.5 \mathrm{~Hz}$ (Crown et al., 2002). In addition, if the effect of FT stimulation reflects a form of learning, some trade-off would be expected between stimulus number and frequency, with spaced presentation being more effective than massed (Groves et al., 1969; Davis, 1970). From this perspective, higher frequency stimulation may have a restorative effect, but require a greater shock number.

\section{Experiment 4: Evidence of Savings Across Days}

A hallmark of learning is the retention (memory) of information over time. For timing, a learning account assumes that a feature of the training is abstracted and stored, yielding some savings across days. From Experiment 1, we know that the emergence of the restorative effect requires more than 360 shocks. Here we assessed whether FT stimulation has a restorative effect when the additional (360) shocks are given $24 \mathrm{~h}$ later. We also tested whether it matters if the stimuli occur to the same, or a different, dermatome (tail vs. leg). If the FT effect reflects a form of spinal learning, that is centrally mediated, it should emerge when FT stimulation is given across days to distinct dermatomes.

The experimental design is illustrated at the top of Figure 4. A day after spinal transection, two groups received 360 FT shocks at $0.5 \mathrm{~Hz}$ to the tail or leg. The next day, half of the subjects (720 Same Locus) received $360 \mathrm{FT}$ shocks at $0.5 \mathrm{~Hz}$ to the same location $(n=8)$. The remaining subjects $(n=8)$ received shock to the other dermatome (720 Different Locus). A third group (Unshocked) was restrained, and had the electrodes attached on both days, but received no shock $(n=8)$. Subjects in a fourth group (360 Once) were also restrained on both 


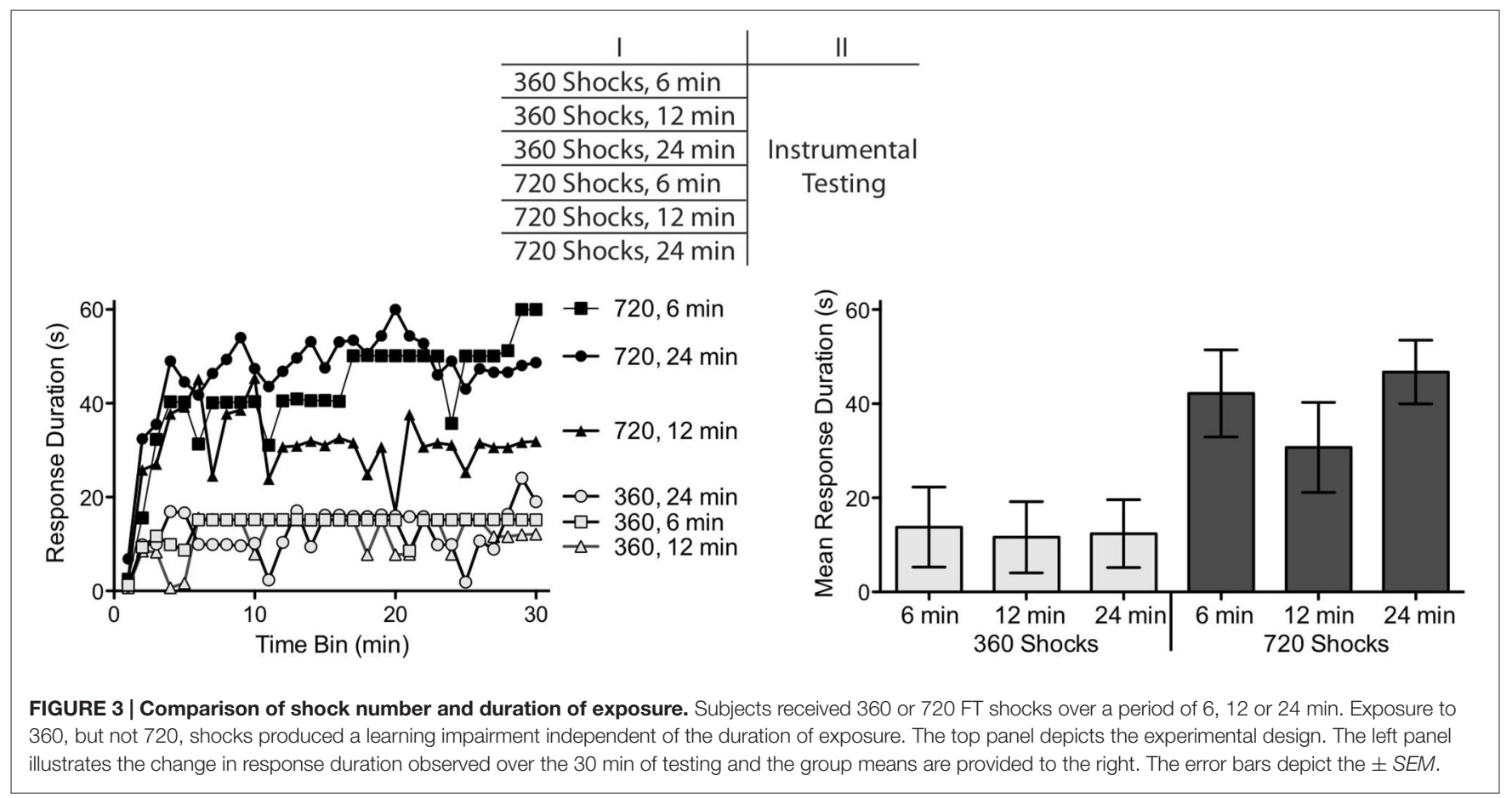

days, but received just one bout of $360 \mathrm{FT}$ shocks at $0.5 \mathrm{~Hz}$. Half of these subjects $(n=4)$ were given shock on the first day while the remaining subjects $(n=4)$ received shock the next day. The site of shock treatment was counter-balanced across subjects. Immediately after the second shock treatment, instrumental learning was tested on the untreated hindleg as described above.

From the results of the previous experiments, we expected that subjects given just 360 fixed spaced shocks would exhibit a learning impairment independent of whether the stimuli occurred immediately before testing or $24 \mathrm{~h}$ earlier. Both treatments did, indeed, produce similar results, $F_{(1,6)}<1.0$, $p>0.05$. Given this, we collapsed the results across the two conditions to form a single condition (360 Once).

As shown in Figure 4, the Unshocked group learned whereas rats given a single session of 360 FT shocks (360 Once) did not. Most importantly, a second bout of 360 shocks given 24 h later reinstated the capacity to learn and this was true independent of whether the shocks were presented to the same (720 Same Locus) or different (720 Different Locus) dermatomes. An ANOVA revealed a significant main effect of shock condition, and a significant Time $\times$ Shock Treatment interaction $F^{\prime} s>5.23, p<$ 0.05. Post hoc comparisons of the group means confirmed that the 360 Once group differed from all other groups $(p<0.05)$. No other differences approached significance $(p>0.05)$.

Subjects that received only 360 shocks exhibited a learning impairment. Exposure to additional FT stimulation reinstated the capacity to learn. Because the treatments were separated by $24 \mathrm{~h}$, this implies that the initial bout of stimulation had an enduring effect, inducing a kind of memory that spanned the gap in time. Further, because this effect emerged when shocks were presented to distinct dermatomes, the results imply a form of spatial integration that must depend upon a central (spinal) system.

\section{Experiment 5: Spinal Neurons Encode an Index of Regularity, not the Specific Interval of Time}

We have shown that two bouts of 360 FT shocks, separated by 24 $\mathrm{h}$, can produce the FT effect. The implication is that a feature related to regularity is abstracted and stored during the initial bout of 360 shocks. One possibility is that spinal systems encode the particular interval $(2 \mathrm{~s})$ that separated the shocks, a process that we will refer to as hard timing. An alternative possibility is that spinal systems just encode that the incoming stimulus was regular in nature (an index of regularity). From this view, what matters is that both bouts are regular, not whether the ISI matches. We refer to this alternative as soft timing. To evaluate these alternatives, we again exposed subjects to two bouts of 360 shocks, separated by $24 \mathrm{~h}$. However, in this experiment, the shocks within each bout were spaced either $0.5 \mathrm{~s}(2 \mathrm{~Hz})$ or $2 \mathrm{~s}(0.5 \mathrm{~Hz})$ apart. One group received shock at the same frequency across days $(720 \mathrm{Same} \mathrm{Hz})$ while another received shock at different frequencies (720 Different $\mathrm{Hz}$ ). A third group (360 Once) received just a single bout of shock, at an ISI of 0.5 or $2 \mathrm{~s}$, which should induce a learning impairment.

We conducted this experiment in two replications $(n=6)$ and used 48 rats. Because similar results were obtained across each replication, $F_{(2,30)}=1.03, p>0.05$, we collapsed the data across this variable. The experimental design is illustrated at the top of Figure 5. As in Experiment 4, subjects received two training sessions, beginning a day after surgery. The second session occurred $24 \mathrm{~h}$ after session 1 . One group received 360 shocks 

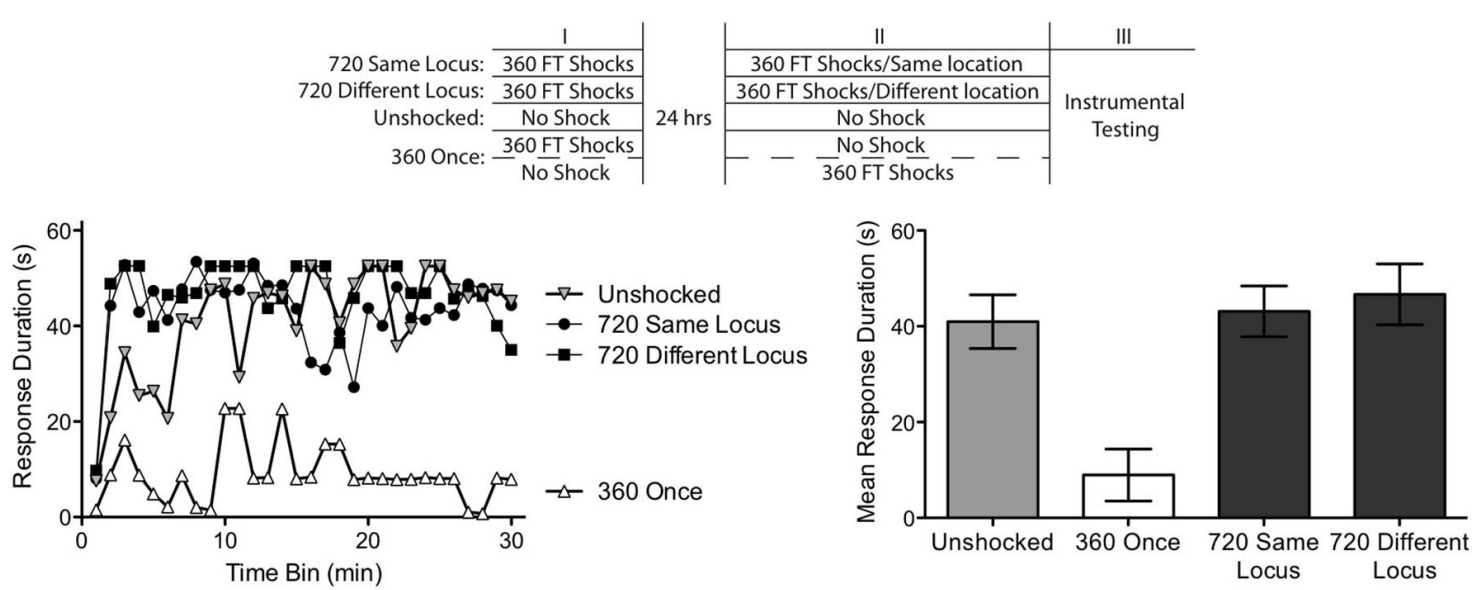

FIGURE 4 | Abstraction of regularity over time and shock locus. Subjects received two shock treatments, starting a day after a spinal transection. One group (720 Same Locus) received 360 FT shocks on each day to the same location (the tail or leg). Another (720 Different Locus) received 360 FT shocks on each day, but on different dermatomes. A third group never received shock (Unshocked). The last group (360 Once) received a single bout of 360 FT shocks, either during the first or second session. The experimental design is depicted in the top panel. All subjects were then tested for 30 min with response-contingent shock applied to the untreated leg. As expected, unshocked rats exhibited an increase in response duration over the 30 min of testing (left panel). Exposure to a single bout of $360 \mathrm{FT}$ shocks induced a learning impairment. Two bouts of FT shock eliminated the learning impairment and this was true independent of whether the shocks were given at the same or different locations across days. Mean performance, $\pm S E M$, is illustrated in the right panel.

on both days at the same frequency, either 0.5 of $2 \mathrm{~Hz}$. For both conditions, subjects were restrained an equivalent period of time and shocks were presented to different dermatomes (leg or tail; with both stimulus frequency and dermatome counter-balanced across subjects). Because both conditions yielded similar results, $F_{(1,10)}<1.0, p>0.05$, we collapsed the data across these two conditions to form a single group (720 Same Hz). Other rats were treated the same but received shock at different frequencies across days (720 Different $\mathrm{Hz}$ ). Again, both stimulation site and frequency were counter-balanced across days. A third group received a single bout of 360 shocks, given at an ISI of 0.5 or $2 \mathrm{~s}$ on Day 1 or 2 . Because similar results were obtained across dermatome, ISI, and day, the data were collapsed to form a single group (360 Once). Finally, a fourth group was restrained an equivalent period of time, but remained unshocked. After the second treatment, subjects underwent $30 \mathrm{~min}$ of instrumental testing as described above.

Subjects given a single session of 360 shocks (360 Once) exhibited impaired learning (Figure 5). A second session of fixed spaced shock had a restorative effect independent of whether it was presented at the same or different frequency. An ANOVA confirmed that shock treatment had a significant effect, $F_{(2,33)}=$ $5.40, p<0.01$. There was also a significant effect of time and Time $\times$ Shock Treatment interaction, both $F^{\prime}$ s $>1.72, p<0.05$. Post hoc comparisons of the group means showed that the groups that received one session of FT stimulation differed from the other two $(p<0.05)$. No other comparisons were significant $(p>0.05)$.

Our results provide further evidence of a savings effect, demonstrating that two bouts of regular stimulation can induce the FT effect when separated by $24 \mathrm{~h}$. At issue was the nature of the memory laid down by the initial bout of stimulation. Do spinal systems abstract the specific interval between stimuli (hard timing) or simply an index of regularity (soft timing). Our results support the latter conclusion because a similar outcome was obtained irrespective of whether the shocks presented on Day 1 and 2 had the same or different frequencies.

\section{DISCUSSION}

Prior work had shown that the effect of fixed spaced shock varies as a function of shock number, with 180 shocks producing a learning impairment and 720 having a restorative effect (Baumbauer et al., 2008, 2009). Here we derived the shock number needed to transform the effect of stimulation, showing that a restorative effect emerges after 540 shocks (Experiment 1 ). We then showed that the effect of FT and VT stimulation remains stable if additional shocks $(4,500)$ are given (Experiment 2 ). When 360 versus $720 \mathrm{FT}$ shocks were given over 6, 12, or $24 \mathrm{~min}$, we found that 360 shocks consistently produced a learning impairment whereas 720 did not (Experiment 3). This implies that the critical factor is shock number, not the duration of exposure. We then used this information to explore the nature of what is learned. It was assumed here that learning involves an incremental process that develops over the course of training. If this is true, an initial bout of FT stimulation (insufficient to restore learning) should have a lasting effect that fosters the development of the restorative effect when training is continued. We explored this possibility by administering two bouts of 360 FT shocks separated by 24 hrs (Experiment 4). As expected, a single bout of 360 shocks induced a learning impairment. When combined, the capacity for learning was restored, demonstrating a form of savings. Moreover, two bouts of shock yielded a restorative effect when applied to distinct dermatomes, implying a kind of central (spinal) integration. We then used this savings effect to explore the nature of the underlying memory. Here we introduced a distinction between hard and soft timing, suggesting that the former involves a memory for the temporal interval whereas the latter simply 

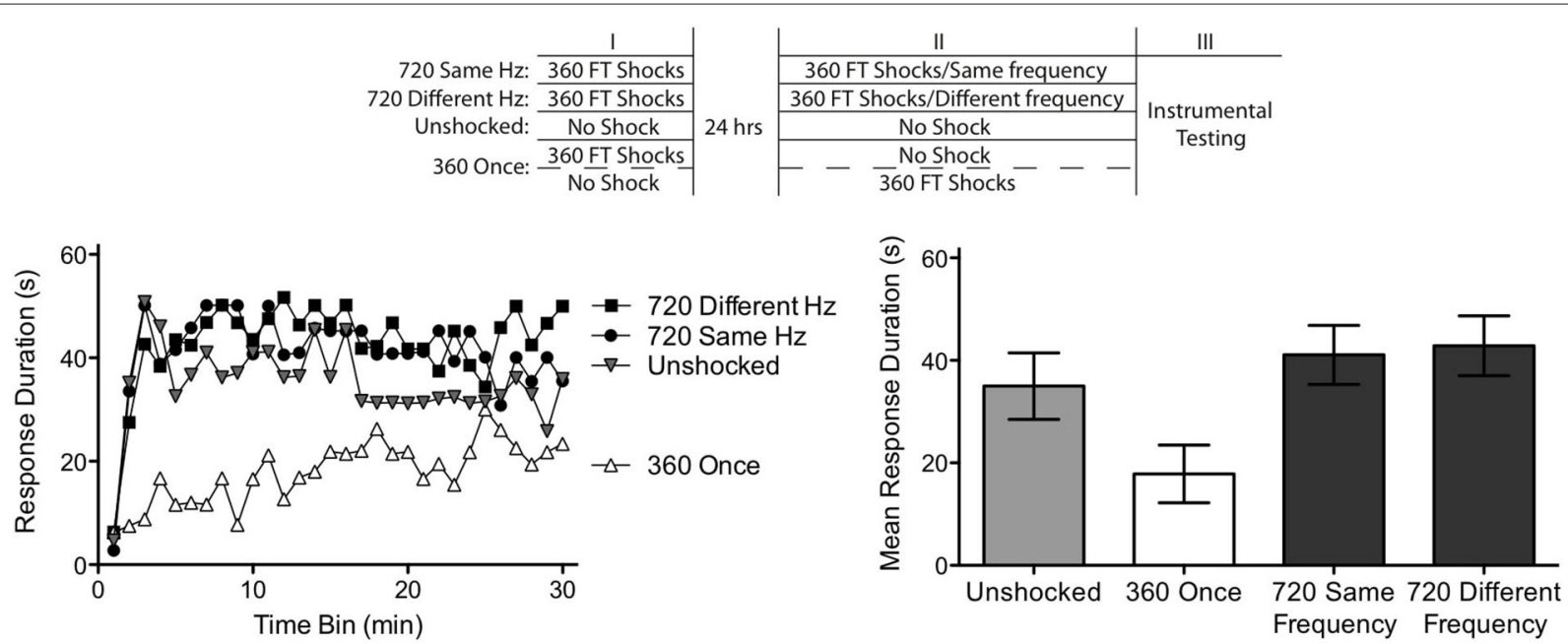

FIGURE 5 | Abstraction of regularity over time and shock frequency. Subjects received two shock treatments, starting a day after a spinal transection. One group (720 Same Hz) received $360 \mathrm{FT}$ shocks on each day at the same frequency ( $0.5 \mathrm{or} 2 \mathrm{~Hz}$ ). Another (720 Different Hz) received $360 \mathrm{FT}$ shocks on each day, but on different frequencies. A third group never received shock (Unshocked). The last group (360 Once) received a single bout of $360 \mathrm{FT}$ shocks, during either the first or second session. Half of these subjects received shock at $0.5 \mathrm{~Hz}$ while the remaining received shock at $2 \mathrm{~Hz}$. This experimental design is detailed in the top panel. Subjects were then tested for 30 min with response-contingent shock applied to the untreated leg. As expected, unshocked rats exhibited an increase in response duration over the $30 \mathrm{~min}$ of testing (left panel). Exposure to a single bout of $360 \mathrm{FT}$ shocks induced a learning impairment. Two bouts of $\mathrm{FT}$ shock eliminated the learning impairment and this was true independent of whether the shocks were given at the same or different frequencies across days. Mean performance, \pm SEM, is illustrated in the right panel.

encodes that there was a period of regular stimulation. We explored this issue by applying two bouts of 360 FT shock and varying the frequency of stimulation. We found that FT stimulation had a restorative effect independent of whether the frequency of stimulation across bouts remained the same or differed (Experiment 5). This implies that spinal systems do not store the specific interval between shocks but rather encode that there was a period of regular stimulation.

Elsewhere, we have explored the mechanism that underlies the abstraction of regularity within the spinal cord (Lee et al., submitted). We reasoned that this could be accomplished by means of either an internal oscillator (a process that exhibits a pendulum-like regularity over time) or a timer (an hourglasslike device that decays over a fixed period). If an oscillator is at work, the occasional omission of a stimulus may have little effect; as long as subsequent shocks are presented in phase, the process could continue on at the same tempo. Supporting this, we found that randomly omitting up to half the shocks from a train of FT stimulation has no impact on the development of the restorative effect, provided the shocks remain in phase (Lee et al., submitted). In addition, we have observed that when FT stimulation is applied in combination with a drug cocktail that promotes spinally-mediated motor behavior (Strain et al., 2014), it can engage a rhythmic swinging of a the tail (pendulate tail) that occurs at the same frequency as the eliciting stimulus and continues for minutes after stimulation has ended (Strain et al., 2014). These observations suggest that regular stimulation engages a neural oscillator.

It has been recognized for many years that the lumbosacral spinal cord contains oscillators (central pattern generators, CPGs) that drive rhythmic behavior (e.g., stepping, scratching;
Brown, 1911; Grillner, 1973; Grillner and Zangger, 1979; Rossignol et al., 1996, 2009; Kiehn and Kjaerulff, 1998; Marder and Bucher, 2001; Kiehn, 2006; Hultborn and Nielsen, 2007; Guertin, 2009; Rossignol and Frigon, 2011; Frigon, 2012). Research has further shown that the neural machinery needed to organize the pattern of leg movements lies within the lower lumbosacral (L3-S2) tissue (Cazalets et al., 1995; Magnuson et al., 1999, 2005), the same region required for spinally mediated instrumental learning (Liu et al., 2005). Interestingly, the neural system that sets the tempo of stepping is located rostrally, within the $\mathrm{L} 1-\mathrm{L} 2$ region. We have suggested that this CPG may mediate the abstraction of regularity. Supporting this, we have shown that surgically disconnecting the L1-L2 region from the lower lumbar spinal cord transforms how FT stimulation affects spinal function; when surgically disconnected from the L1-L2 region, an extended exposure to FT shock has a VT-like effect and induces a learning impairment (Lee et al., submitted).

These observations suggest that spinal timing depends upon the same neural systems that drive the tempo of stepping. The current work shows that engaging this oscillatory mechanism lays down a kind of memory that is preserved across days, allowing two sub-threshold bouts of stimulation to have an additive effect. What appears to underlie this cumulative effect is a process linked to the abstraction of regularity (e.g., that a neural oscillator was engaged), not the specific time interval. We further showed that what is important is not the duration that the oscillator is engaged, but rather the number of oscillations (stimuli). Similarly, Cha et al. (2007) found that increasing step number within a training session (from 100 to 1,000) had a lasting beneficial effect, increasing the quality of stepping over a range of treadmill speeds. Likewise, spinally transected animals 
that receive repeated bouts of step training across days exhibit a benefit from training, that restores the capacity to step on a treadmill across a range of treadmill speeds (de Leon et al., 1997, 1998; van de Crommert et al., 1998; Frigon and Gossard, 2009, 2010; van den Brand et al., 2012). Our work suggests that the emergence of these effect depends upon the number of regular steps taken each day rather than the duration of training. From this perspective, step training could allow spinal neurons to abstract a form of regularity, related to treadmill speed and step frequency. The current study implies that, within a training session, these factors should be kept constant (to allow for the abstraction of regularity), but across days different treadmill speeds (step rates) could be used. Introducing variability in treadmill speed within a session could undermine the longterm benefit of training, which may help to explain why some locomotor training paradigms appear less effective.

If step training and FT stimulation promote adaptive plasticity by engaging a common process, then the latter may be used to promote the former. Indeed, rhythmic stimulation to the tail or perineum is routinely used to promote stepping in spinally transected animals (Rossignol et al., 1996, 2009; Rossignol and Frigon, 2011; Alluin et al., 2015). FT stimulation and step training could also benefit adaptive plasticity for a common reason, for both may up-regulate the expression of BDNF (Gómez-Pinilla et al., 2002, 2007; Baumbauer et al., 2009; Huie et al., 2012b). Additionally, many forms of environmental perturbations (obstacles, immobilization, changes in load) that activate proprioceptive and afferent signals had been show to affect CPG output and cause adaptive changes, indicating that the spinal CPG is not only capable of adapting to environmental changes, but that these environmental changes can cause changes in behavior based on a form of temporal processing. Studies focused on the role of afferent feedback in modifying CPG activity have found that though phase durations and transitions are controlled by the spinal CPG, inputs from peripheral mechanoreceptors can alter the timing of the pattern of motor activation (Frigon and Gossard, 2009, 2010). When weak stimuli (simulating cutaneous tactile stimulation) or actual mechanical tactile stimulation was applied to the dorsal surface of the paw during the extension phase, activation of the extension muscles was markedly increased (Forssberg et al., 1975). Additionally, when an obstacle is placed on a treadmill during training after spinal cord injury, an increase in flexion is observed on subsequent steps and this hyperflexion elicited by cutaneous stimulation of the dorsal surface of the paw persists after the obstacle is removed (Nakada et al., 1994). This suggests a form of memory for the obstacle and

\section{REFERENCES}

Alluin, O., Delivet-Mongrain, H., and Rossignol, S. (2015). Inducing hindlimb locomotor activity in adult rat after a complete thoracic spinal cord section using repeated treadmill training with perineal stimulation only. J. Neurophysiol. 114, 1931-1946. doi: 10.1152/jn.00416.2015

Barbeau, H., and Rossignol, S. (1987). Recovery of locomotion after chronic spinalization in the adult cat. Brain Res. 412, 84-95. doi: 10.1016/00068993(87)91442-9 implies that the behavior was timed to the swing phase. These results demonstrate that CPG entrainment can lead to a change in behavior due to learning about environmental stimuli.

Conversely, the adverse effect of VT stimulation appears to share commonalities with the processes that sensitize nociceptive neurons. Supporting this, we have shown that treatments that induce a central sensitization (e.g., peripheral application of the irritant capsaicin) impair instrumental learning (Ferguson et al., 2006; Hook et al., 2008). Further, both VT stimulation and capsaicin induce an enhanced mechanical reactivity (EMR) to von Frey stimuli applied to the plantar surface of the hind paw. These effects have been related to an up-regulation of the cytokine tumor necrosis factor (TNF; Huie et al., 2012a; Garraway et al., 2014). Just as FT stimulation opposes the adverse effect of VT shock, it also prevents and reverses both the learning impairment and EMR induced by capsaicin treatment (Baumbauer and Grau, 2011; Baumbauer et al., 2012). If FT stimulation and locomotor training engage common processes, the latter should also attenuate the development of neuropathic pain. Conversely, to the extent locomotor training involves a form of instrumental learning, we would anticipate that it too would be disrupted by peripheral inflammation. Recent observations support these hypothesized opponent relations (Hutchinson et al., 2004; Bouffard et al., 2014).

Taken together, our results imply that FT stimulation can have therapeutic value. This adds to the growing body of research demonstrating that both cutaneous and epidural stimulation can have a neuromodulatory effect (Harkema et al., 2011; Ferguson et al., 2012; Grau et al., 2014). What we have shown is that the effect of stimulation depends upon both behavioral control and temporal regularity - regular/controllable stimulation appears to promote adaptive plasticity by means of a BDNF-dependent process whereas irregular/unpredictable stimulation induces a form of maladaptive plasticity that inhibits spinal learning and enhances nociceptive reactivity through a TNF-dependent process (Grau et al., 2012, 2014). Our work suggests that these treatments can have a lasting effect. Further work is needed to evaluate the stimulus parameters that promote adaptive plasticity, the neurobiological mechanisms involved, and how these treatments can be translated to humans.

\section{ACKNOWLEDGMENTS}

The authors wish to thank Russell Huie and Melissa Brumley for their input. The work was supported by a grant from the National Institute of Child Health and Development (HD058412).
Barrière, G., Leblond, H., Provencher, J., and Rossignol, S. (2008). Prominent role of the spinal central pattern generator in the recovery of locomotion after partial spinal cord injuries. J. Neurosci. 28, 3976-3987. doi: 10.1523/jneurosci. 5692-07.2008

Baumbauer, K. M., and Grau, J. W. (2011). Timing in the absence of supraspinal input III: regularly spaced cutaneous stimulation prevents and reverses the spinal learning deficit produced by peripheral inflammation. Behav. Neurosci. 125, 37-45. doi: 10.1037/a0 022009 
Baumbauer, K. M., Hoy, K. C., Huie, J. R., Hughes, A. J., Woller, S. A., Puga, D. A., et al. (2008). Timing in the absence of supraspinal input I: variable, but not fixed, spaced stimulation of the sciatic nerve undermines spinallymediated instrumental learning. Neuroscience 155, 1030-1047. doi: 10.1016/j. neuroscience.2008.07.003

Baumbauer, K. M., Huie, J. R., Hughes, A. J., and Grau, J. W. (2009). Timing in the absence of supraspinal input II: regularly spaced stimulation induces a lasting alteration in spinal function that depends on the NMDA receptor, BDNF release and protein synthesis. J. Neurosci. 29, 14383-14393. doi: 10. 1523/jneurosci.3583-09.2009

Baumbauer, K. M., Lee, K. H., Puga, D. A., Woller, S. A., Hughes, A. J., and Grau, J. W. (2012). Temporal regularity determines the impact of electrical stimulation on tactile reactivity and response to capsaicin in spinally transected rats. Neuroscience 227, 119-133. doi: 10.1016/j.neuroscience.2012.09.054

Bélanger, M., Drew, T., Provencher, J., and Rossignol, S. (1996). A comparison of treadmill locomotion in adult cats before and after spinal transection. J. Neurophysiol. 76, 471-491.

Bouffard, J., Bouyer, L. J., Roy, J.-S., and Mecier, C. (2014). Tonic pain experienced during locomotor training impairs retention despite normal performance during acquisition. J. Neurosci. 34, 9190-9195. doi: 10.1523/jneurosci.5303-13. 2014

Brown, T. G. (1911). The intrinsic factors in the act of progression in the mammal. Proc. R. Soc. Lond. Biol. Sci. 84, 308-319. doi: 10.1098/rspb.1911.0077

Cazalets, J. R., Borde, M., and Clarac, F. (1995). Localization and organization of the central pattern generator for hindlimb locomotion in newborn rat. $J$. Neurosci. 15, 4943-4951.

Cha, J., Heng, C., Reinkensmeyer, D. J., Roy, R. R., Edgerton, V. R., and De Leon, R. R. (2007). Locomotor ability in spinal rats is dependent on the amount of activity imposed on the hindlimbs during treadmill training. J. Neurotrauma 24, 1000-1012. doi: 10.1089/neu.2006.0233

Crown, E. D., and Grau, J. W. (2001). Preserving and restoring behavioral potential within the spinal cord using an instrumental training paradigm. J. Neurophysiol. 86, 845-855.

Crown, E. D., Joynes, R. L., Ferguson, A. R., and Grau, J. W. (2002). Instrumental learning within the spinal cord: IV. Induction and retention of the behavioral deficit observed after noncontingent shock. Behav. Neurosci. 116, 1032-1051. doi: 10.1037/0735-7044.116.6.1032

Davis, M. (1970). Effects of ISI length and variability on startle response habituation to different intensities of tone. Psychonom. Sci. 12, 337-338.

de Leon, R. D., Hodgson, J. A., Roy, R. R., and Edgerton, V. R. (1997). Locomotor capacity attributable to step training versus spontaneous recovery after spinalization in adult cats. J. Neurophysiol. 79, 1329-1340.

de Leon, R. D., Hodgson, J. A., Roy, R. R., and Edgerton, V. R. (1998). Full weightbearing hindlimb standing following stand training in the adult spinal cat. $J$. Neurophysiol. 80, 83-91.

Edgerton, V. R., Tillakaratne, N. J., Bigbee, A. J., de Leon, R. D., and Roy, R. R. (2004). Plasticity of the spinal neural circuitry after injury. Annu. Rev. Neurosci. 27, 145-167. doi: 10.1146/annurev.neuro.27.070203.144308

Ferguson, A. R., Crown, E. D., and Grau, J. W. (2006). Nociceptive plasticity inhibits adaptive learning in the spinal cord. Neuroscience 141, 421-431. doi: 10. 1016/j.neuroscience.2006.03.029

Ferguson, A. R., Huie, J. R., Crown, E. D., Baumbauer, K. M., Hook, M. A., Garraway, S. M., et al. (2012). Maladaptive spinal plasticity opposes adaptive spinal learning after spinal cord injury. Front. Physiol. 3:399. doi: 10.3389/fphys. 2012.00399

Forssberg, H., Grillner, S., and Rossignol, S. (1975). Phase dependent reflex reversal during walking in chronic spinal cats. Brain Res. 85, 103-107. doi: 10. 1016/0006-8993(75)91013-6

Frigon, A. (2012). Central pattern generators of the mammalian spinal cord. Neuroscientist 18, 56-69. doi: 10.1177/1073858410396101

Frigon, A., and Gossard, J. P. (2009). Asymmetric control of cycle period by the spinal locomotor rhythm generator in the adult cat. J. Physiol. 587, 4617-4628. doi: 10.1113/jphysiol.2009.176669

Frigon, A., and Gossard, J. P. (2010). Evidence for specialized rhythm-generating mechanisms in the adult mammalian spinal cord. J. Neurosci. 30, 7061-7071. doi: 10.1523/jneurosci.0450-10.2010

Garraway, S. M., Woller, S. A., Huie, J. R., Hartman, J. J., Hook, M. A., Miranda, R. C., et al. (2014). Peripheral noxious stimulation reduces withdrawal threshold to mechanical stimuli after spinal cord injury: role of tumor necrosis factor alpha and apoptosis. Pain 155, 2344-2359. doi: 10.1016/j.pain.2014. 08.034

Gómez-Pinilla, F., Huie, J. R., Ying, Z., Ferguson, A., Crown, E. D., Baumbauer, K. M., et al. (2007). BDNF and learning: evidence that instrumental training promotes learning within the spinal cord by up-regulating BDNF expression. Neuroscience 148, 893-906. doi: 10.1016/j.neuroscience.2007.05.051

Gómez-Pinilla, F., Ying, Z., Roy, R. R., Molteni, R., and Edgerton, V. R. (2002). Voluntary exercise induces a BDNF-mediated mechanism that promotes neuroplasticity. J. Neurophysiol. 88, 2187-2195. doi: 10.1152/jn.00152.2002

Grau, J. W. (2014). Learning from the spinal cord: how the study of spinal cord plasticity informs our view of learning. Neurobiol. Learn. Mem. 108, 155-171. doi: 10.1016/j.nlm.2013.08.003

Grau, J. W., Barstow, D. G., and Joynes, R. L. (1998). Instrumental learning within the spinal cord: I. Behavioral properties. Behav. Neurosci. 112, 1366-1386. doi: 10.1037/0735-7044.112.6.1366

Grau, J. W., Crown, E. D., Ferguson, A. R., Washburn, S. N., Hook, M. A., and Miranda, R. C. (2006). Instrumental learning within the spinal cord: underlying mechanisms and implications for recovery after injury. Behav. Cogn. Neurosci. Rev. 5, 191-239. doi: 10.1177/1534582306289738

Grau, J. W., Huie, J. R., Garraway, S. M., Hook, M. A., Crown, E. D., Baumbauer, K. M., et al. (2012). Impact of behavioral control on the processing of nociceptive stimulation. Front. Physiol. 3:262. doi: 10.3389/fphys.2012.00262

Grau, J. W., Huie, J. R., Lee, K. H., Hoy, K. C., Huan, Y.-J., Turtle, J. D., et al. (2014). Metaplasticity and behavior: how training and inflammation affect plastic potential within the spinal cord and recovery after injury. Front. Neural Circuits 8:100. doi: 10.3389/fncir.2014.00100

Grau, J. W., Washburn, S. N., Hook, M. A., Ferguson, A. R., Crown, E. D., Garcia, G., et al. (2004). Uncontrollable nociceptive stimu- lation undermines recovery after spinal cord injury. J. Neurotrauma 21, 1795-1817. doi: 10. 1089/0897715042664948

Grillner, S. (1973). "Locomotion in the spinal cat," in Control of Posture and Locomotion, eds R. B. Stein, K. G. Pearson, R. S. Smith, and J. B. Redford (New York, NY: Plenum Press), 515-535.

Grillner, S., and Zangger, P. (1979). On the central generation of locomotion in the low spinal cat. Exp. Brain Res. 34, 241-261. doi: 10.1007/bf00235671

Groves, P. M., Lee, D., and Thompson, R. F. (1969). Effects of stimulus frequency and intensity on habituation and sensitization in acute spinal cat. Physiol. Behav. 4, 383-388. doi: 10.1016/0031-9384(69)90194-2

Guertin, P. A. (2009). The mammalian central pattern generator for locomotion. Brain Res. Rev. 62, 45-56. doi: 10.1016/j.brainresrev.2009.08.002

Harkema, S., Gerasimenko, Y., Hodes, J., Burdick, J., Angeli, C., Chen, Y., et al. (2011). Effect of epidural stimulation of the lumbosacral spinal cord on voluntary movement, standing and assisted stepping after motor complete paraplegia: a case study. Lancet 377, 1938-1947. doi: 10.1016/s01406736(11)60547-3

Hook, M. A., Huie, J. R., and Grau, J. W. (2008). Peripheral inflammation undermines the plasticity of the isolated spinal cord. Behav. Neurosci. 122, 233-249. doi: 10.1037/0735-7044.122.1.233

Huie, J. R., Baumbauer, K. M., Lee, K. H., Beattie, M. S., Bresnahan, J. C., Ferguson, A. R., et al. (2012a). Glial tumor necrosis factor alpha (TNF $\alpha$ ) generates metaplastic inhibition of spinal learning. PLoS One 7:e39751. doi: 10 1371/journal.pone.0039751

Huie, J. R., Garraway, S. M., Baumbauer, K. M., Hoy, K. C., Beas, B. S., Montgomery, K. S., et al. (2012b). Brain-derived neurotrophic factor promotes adaptive plasticity within the spinal cord and mediates the beneficial effects of controllable stimulation. Neuroscience 200, 74-90. doi: 10.1016/j.neuroscience. 2011.10.028

Hultborn, H., and Nielsen, J. B. (2007). Spinal control of locomotion - from cat to man. Acta Physiol. (Oxf) 189, 111-121. doi: 10.1111/j.1748-1716.2006.01651.x

Hutchinson, K. J., Gomez-Pinilla, F., Crowe, M. J., Ying, Z., and Basso, D. M. (2004). Three exercise paradigms differentially improve sensory recovery after spinal cord contusion in rats. Brain 127, 1403-1414. doi: 10.1093/brain/awh160

Ji, R.-R., Kohno, T., Moore, K. A., and Woolf, C. J. (2003). Central sensitization and LTP: do pain and memory share similar mechanisms? Trends Neurosci. 26, 696-705. doi: 10.1016/j.tins.2003.09.017

Kiehn, O. (2006). Locomotor circuits in the mammalian spinal cord. Annu. Rev. Neurosci. 29, 279-306. doi: 10.1146/annurev.neuro.29.051605.112910 
Kiehn, O., and Kjaerulff, O. (1998). Distribution of central pattern generators for rhythmic motor outputs in the spinal cord of limbed vertebrates. Ann. NY Acad. Sci. 860, 110-129. doi: 10.1111/j.1749-6632.1998.tb09043.x

Liu, G. T., Ferguson, A. R., Crown, E. D., Bopp, A. C., Miranda, R. C., and Grau, J. W. (2005). Instrumental learning within the rat spinal cord: localization of the essential neural circuit. Behav. Neurosci. 119, 538-547. doi: 10.1037/0735-7044. 119.2.538

Magnuson, D., Lovett, R., Coffee, C., Gray, R., Han, Y., Zhang, P., et al. (2005). Functional consequences of lumbar spinal cord contusion injuries in the adult rat. J. Neurotrauma 22, 529-543. doi: 10.1089/neu.2005. 22.529

Magnuson, D. S., Trinder, T. C., Zhang, Y. P., Burke, D., Morassutti, D. J., and Shields, C. B. (1999). Comparing deficits following excitotoxic and contusion injuries in the thoracic and lumbar spinal cord of the adult rat. Exp. Neurol. 156, 191-204. doi: 10.1006/exnr.1999.7016

Marder, E., and Bucher, D. (2001). Central pattern generators and the control of rhythmic movements. Curr. Biol. 11, R986-R996. doi: 10.1016/s09609822(01)00581-4

Nakada, K., Hodgson, J. A., de Leon, R. D., Roy, R. R., and Edgerton, V. R. (1994). Prolonged modification of the mechanisms of the step cycle by single and repetitive mechanical stimuli in chronic spinal cats. Soc. Neurosci. Abstr. 20:1755.

Rossignol, S., Barrière, G., Alluin, O., and Frigon, A. (2009). Re-expression of locomotor function after partial spinal cord injury. Physiology (Bethesda) 24, 127-139. doi: 10.1152/physiol.00042.2008

Rossignol, S., Chau, C., Brustein, E., Bélanger, M., Barbeau, H., and Drew, T. (1996). Locomotor capacities after complete and partial lesions of the spinal cord. Acta Neurobiol. Exp. (Wars) 56, 449-463.

Rossignol, S., and Frigon, A. (2011). Recovery of locomotion after spinal cord injury: some facts and mechanisms. Annu. Rev. Neurosci. 34, 413-440. doi: 10. 1146/annurev-neuro-061010-113746

Sandkühler, J. (2000). Learning and memory in pain pathways. Pain 88, 113-118. doi: 10.1016/s0304-3959(00)00424-3
Strain, M., Pesek, B., Huang, Y., Stump, C., Turtle, J., Lee, K., et al. (2014). "Acquired pendulate tail: evidence for the activation of an oscillator following fixed spaced shock," in Program No. 115.06. 2014 Neuroscience Meeting Planner (Washington, DC: Society for Neuroscience, 2014).

van de Crommert, H. W., Mulder, H. W., and Duysens, J. (1998). Neural control of locomotion: sensory control of the central pattern generator and its relation to treadmill training. Gait Posture 7, 251-263. doi: 10.1016/s0966-6362(98) 00010-1

van den Brand, R., Heutschi, J., Barraud, Q., DiGiovanna, J., Bartholdi, K., Huerlimann, M., et al. (2012). Restoring voluntary control of locomotion after paralyzing spinal cord injury. Science 336, 1182-1185. doi: 10.1126/science. 1217416

Willis, W. D. (2001). "Mechanisms of central sensitization of nociceptive dorsal horn neurons," in Spinal Cord Plasticity: Alterations in Reflex Function, eds M. M. Patterson and J. W. Grau (Norwell, MA: Kluwer Academic Publishers), $127-162$.

Woolf, C. J., and Thompson, S. W. N. (1991). The induction and maintenance of central sensitization is dependent on N-methyl-D-aspartic acid receptor activation; implications for the treatment of post-injury pain hypersensitivity states. Pain 44, 293-299. doi: 10.1016/0304-3959(91) 90100-c

Conflict of Interest Statement: The authors declare that the research was conducted in the absence of any commercial or financial relationships that could be construed as a potential conflict of interest.

Copyright (c) 2015 Lee, Turtle, Huang, Strain, Baumbauer and Grau. This is an open-access article distributed under the terms of the Creative Commons Attribution License (CC BY). The use, distribution and reproduction in other forums is permitted, provided the original author(s) or licensor are credited and that the original publication in this journal is cited, in accordance with accepted academic practice. No use, distribution or reproduction is permitted which does not comply with these terms. 